\title{
Molecular Surveillance and in vitro Drug Sensitivity Study of Plasmodium falciparum Isolates from the China-Myanmar Border
}

\author{
Siqi Wang, ${ }^{1}$ Shiling Xu, ${ }^{1}$ Jinting Geng, ${ }^{1}$ Yu Si, ${ }^{1}$ Hui Zhao, ${ }^{1}$ Xinxin Li, ${ }^{1}$ Qi Yang, ${ }^{1}$ Weilin Zeng,,${ }^{1}$ Zheng Xiang, ${ }^{1}$ Xi Chen, ${ }^{1}$ \\ Yanmei Zhang, ${ }^{1}$ Cuiying Li, ${ }^{1}$ Myat Phone Kyaw, ${ }^{2}$ Liwang Cui, ${ }^{3 *} \dagger$ and Zhaoqing Yang ${ }^{1 *} \dagger$ \\ ${ }^{1}$ Department of Pathogen Biology and Immunology, Kunming Medical University, Kunming, China; ${ }^{2}$ Myanmar Health Network Organization, \\ Yangon, Myanmar; ${ }^{3}$ Department of Internal Medicine, Morsani College of Medicine, University of South Florida, Tampa, Florida
}

\begin{abstract}
The emergence and spread of resistance in Plasmodium falciparum to the frontline treatment artemisininbased combination therapies in Southeast Asia require close monitoring of the situation. Here, we collected 36 clinical samples of $P$. falciparum from the China-Myanmar border in 2014-2016, adapted these parasites to continuous culture, and performed in vitro drug assays on seven antimalarial drugs. Data for 23 parasites collected in 2010 and 2012 from the same area reported in an early study were used to assess longitudinal changes in drug sensitivity. Parasites remained highly resistant to chloroquine (CQ) and pyrimethamine, whereas they were generally sensitive to mefloquine (MFQ), lumefantrine (LMF), naphthoquine (NQ), and pyronaridine (PND). Parasites showed a similar temporal trend in sensitivity to $C Q, N Q$, and $P N D$, with gradual reduction in the half-maximal inhibitory concentrations $\left(I_{50} S\right)$ after 2012 . The $I C_{50} s$ to the aminoalcohol drugs MFQ, LMF, and quinine (QN) all significantly declined in 2014, followed by various degrees of increase in 2016. Pyrimethamine displayed a continuous increase in $\mathrm{IC}_{50}$ over the years. The Dd2-like $P$. falciparum chloroquineresistant transporter mutations were fixed or nearly fixed in the parasite population. The $P$. falciparum multidrug resistance $1 \mathrm{~F} 1226 \mathrm{Y}$ mutation was detected in $80 \%$ parasites in 2016 and associated with reduced sensitivity to LMF and QN $(P<$ 0.05). The N51I in P. falciparum dihydrofolate reductase and K540E/N and A581G in P. falciparum dihydropteroate synthase that are associated with antifolate resistance were either fixed or were approaching fixation in recent years. This study provides an updated picture and temporal trend of antimalarial drug resistance in the China-Myanmar border region, which will serve as a reference for antimalarial treatment.
\end{abstract}

\section{INTRODUCTION}

Malaria is one of the most devastating vector-borne diseases and still threatens the lives of hundreds of millions of people in tropical and subtropical countries. Despite the significant decline in global malaria incidence, there were still 228 million malaria cases in 2018. ${ }^{1}$ The Greater Mekong Subregion (GMS) in Southeast Asia, consisting of Cambodia, Vietnam, Laos, Thailand, Myanmar, and China's Yunnan and Guangxi provinces, has made a great stride in malaria control and is pursuing regional malaria elimination with an aim to achieve this goal by 2030 . China is the first in this region to achieve zero indigenous malaria cases in 2017 , but imported malaria, especially through its porous international borders with other countries in the GMS, threatens the maintenance of the malaria-free status. ${ }^{2}$ The GMS is an epicenter of antimalarial drug resistance, and the emergence of artemisinin resistance in Plasmodium falciparum in the last decade poses a great challenge to malaria elimination. ${ }^{3}$ Artemisinin resistance, first detected in western Cambodia, ${ }^{4,5}$ has been found in most parts of the GMS ${ }^{6-9}$ Artemisinin resistance, together with resistance to the partner drugs, has led to increased clinical failures of artemisinin combination therapies (ACTs) such as artesunate-mefloquine (AS-MFQ) and dihydroartemisininpiperaquine (DHA-PPQ). ${ }^{10-14}$ There is an urgent need to strengthen resistance surveillance on $P$. falciparum, prevent the spread of resistant strains, and implement effective

*Address correspondence to Liwang Cui, Department of Internal Medicine, Morsani College of Medicine, University of South Florida, 3720 Spectrum Blvd., Suite 304, Tampa, FL, 33612. E-mail: liwangcui@usf.edu or Zhaoqing Yang, Department of Pathogen Biology and Immunology, Kunming Medical University, 1168 West Chunrong Rd., Kunming, Yunnan Province, 650500, China. E-mail: zhaoqingy92@hotmail.com

†These authors contributed equally to this work. treatments to ensure timely achievement of malaria elimination.

Drug resistance in malaria parasites is monitored through clinical efficacy studies of frontline drugs, ex vivo or in vitro drug assays, and molecular epidemiological studies of markers associated with resistance. Each method has its own pros and cons, and in combination, they offer a comprehensive picture of antimalarial drug resistance in a region. Although molecular markers are convenient for monitoring the evolution of drug resistance, the resistance mechanisms for many drugs are not understood. Mutations in the $P$. falciparum chloroquine-resistant transporter (pfcrt) are the major determinants of chloroquine (CQ) resistance. ${ }^{15,16} \mathrm{Re}$ cently, some new pfcrt mutations in the GMS were associated with resistance to $P P Q$, another 4-aminoquinoline drug. ${ }^{17,18}$ The $P$. falciparum multidrug resistance 1 gene (pfmdr1) is associated with resistance to multiple antimalarial drugs. ${ }^{19}$ In particular, the N86Y mutation contributes to the enhancement of $\mathrm{CQ}$ resistance. Plasmodium falciparum multidrug resistance 1 amplification has been associated with resistance to mefloquine (MFQ) and artemisinin derivatives in vitro. ${ }^{20}$ The mechanisms of resistance to the antifolate drugs sulfadoxine and pyrimethamine (PY) are well understood and associated with mutations in the $P$. falciparum dihydropteroate synthase (pfdhps) gene (e.g., S436A, A437G, K540E, A581G, and A613S) and $P$. falciparum dihydrofolate reductase (pfdhfr) gene (e.g., N51I, C59R, S108N, and 1164L), respectively. Mutation combinations in the pfdhps and pfdhfr genes mediate the failures of the drug sulfadoxine-PY. ${ }^{21}$ Recent studies have revealed that mutations in the propeller domain of the $P$. falciparum Kelch 13 (Pfk13) gene are associated with artemisinin resistance, which is manifested as delayed parasite clearance. ${ }^{22}$ A global map of $P f k 13$ mutations showed that resistance-conferring mutations were restricted to the GMS. ${ }^{23}$ The recently observed resistance to $P P Q$ in Cambodia was 
associated with gene amplification of the hemoglobin digestive enzyme plasmepsin $2 / 3 .^{24,25}$

To track the evolution of drug resistance in malaria parasites in eastern and northeastern Myanmar along the China-Myanmar border, we collected longitudinal clinical samples from this region and performed in vitro drug assays and molecular studies on the archived parasite samples. ${ }^{26}$ Our earlier studies demonstrated high prevalence of resistance to $\mathrm{CQ}$ and antifolate drugs, despite these drugs have been withdrawn from treating $P$. falciparum for a long time. For several drugs, our studies also revealed significant variations in sensitivity between years. As a continuation of the resistance monitoring efforts, we collected $P$. falciparum clinical isolates in 2014 and 2016 and adapted them to continuous culture. Through in vitro drug assays and genotyping of drug resistance-related genes, we wanted to obtain an updated drug sensitivity profile and understand how the parasite population evolves in the face of intensified control efforts.

\section{MATERIALS AND METHODS}

Parasite sample collection. Clinical isolates of $P$. falciparum were collected in 2014 and 2016 from malaria clinics located at the Nabang township in west Yunnan Province, China, and the Laiza town, Kachin State, Myanmar, along the ChinaMyanmar border. Diagnosis by microscopy was performed initially by field medical staff and later, confirmed by PCR in the laboratory. Patients, after signing the written informed consent, donated up to $5 \mathrm{~mL}$ of venous blood, which was used to establish continuous parasite culture. The human subject protocol for this study was approved by the Institutional Review Boards of Kunming Medical University and Pennsylvania State University.

Genetic polymorphisms of drug resistance genes. DNA extraction from in vitro-cultured $P$. falciparum blood samples was performed by using the High Pure PCR Template Preparation Kit (Roche Life Science, Indianapolis, IN). Parasites were first genotyped at the $P$. falciparum merozoite surface proteins $m s p 1$ and $m s p 2$ and glutamate-rich protein (glurp) genes. ${ }^{27}$ Only the monoclonal infections of the $P$. falciparum isolates were used for genotyping drug resistance genes and for in vitro drug assays. Polymorphisms in resistance-related genes were determined by PCR and sequencing of the amplified fragment. ${ }^{28,29}$ The pfcrt fragments covered codons 72-76, 220, 271, 326, 356, and 371; two pfmdr1 fragments covered codons $86,184,1240$, and 1246; a pfdhfr fragment included codons 51, 59, 108, and 164; and two pfdhps fragments contained the codons 436, 540, and 581 .

In vitro culture of $P$. falciparum and drug assays. Parasite isolates were cultured in type $\mathrm{O}^{+}$human red blood cells (RBCs) in a complete medium under an atmosphere of $92 \% \mathrm{~N}_{2}-3 \%$ $\mathrm{O}_{2}-5 \% \mathrm{CO}_{2}$. Parasites in the ring stage were synchronized by $5 \%$ sorbitol and diluted with complete medium to a $0.5 \%$ parasitemia and $2 \%$ hematocrit. We used the SYBR $®$ green I-based assay to determine the sensitivities of $P$. falciparum isolates to seven antimalarial drugs using a 96-well plate format. Drugs were added to each well of a 96-well microplate at an initial concentration of $3.75 \mu \mathrm{M}$ for $\mathrm{CQ}$ and $\mathrm{PY}, 256 \mathrm{nM}$ for naphthoquine (NQ) and MFQ, $160 \mathrm{nM}$ for pyronaridine (PND), $10.24 \mu \mathrm{M}$ for quinine (QN), and $800 \mathrm{nM}$ for lumefantrine (LMF), which were serially diluted. In each plate, the 3D7 reference strain was included as an internal control. Three technical repeats and two biological replicates were performed for each parasite isolate.

Statistical analyses. All data were analyzed with SPSS 20 and GraphPad Prism 6.0. The half-maximal inhibitory concentrations $\left(\mathrm{IC}_{50} \mathrm{~s}\right)$ were calculated by fitting the drug response data to a sigmoid curve. Geometric mean and SD were calculated based on the replicates of each isolate. Median and interquartile range were used because the data were not normally distributed. The $\mathrm{IC}_{50}$ values between the years were compared using the Mann-Whitney $U$ test. Mutation frequencies of drug resistance genes were analyzed using the Chi-square test.

\section{RESULTS}

In vitro drug sensitivity profiling of parasite isolates. We collected and culture-adapted 16 and 20 monoclonal $P$. falciparum isolates from the China-Myanmar border in 2014 and 2016, respectively. Using a standard SYBR $®$ green-Ibased method, we measured their in vitro $\mathrm{IC}_{50}$ values to seven antimalarials (Figure 1, Table 1). Previously published data of 23 parasite isolates collected in 2010 and 2012 from the same study area were used for constructing the trends. ${ }^{26}$ For $\mathrm{CQ}$, $96.4 \%$ of the parasites were resistant with $\mathrm{IC}_{50}$ values higher than the $100 \mathrm{nM}$ cutoff value (Figure 1). ${ }^{30}$ For MFQ, 42.4\% parasite isolates were considered resistant with $\mathrm{IC}_{50}$ above the $30 \mathrm{nM}$ cutoff value proposed in an earlier study. ${ }^{30}$ The median $\mathrm{IC}_{50}$ of $\mathrm{QN}$ was significantly lower than the cutoff value of $600 \mathrm{nM}$ as proposed earlier, ${ }^{30}$ with $6.8 \%$ of the isolates exceeding this value. For PND, 5.1\% isolates had higher $\mathrm{IC}_{50}$ values than the cutoff value of $15 \mathrm{nM}$ as proposed earlier. ${ }^{31}$ Given that there were no threshold values defined for resistance to LMF and NQ, we used the mean + 2 SDs as arbitrary cutoff values for resistance. ${ }^{32}$ Three isolates had higher $\mathrm{IC}_{50}$ values above the $8.6 \mathrm{nM}$ threshold level for $\mathrm{LMF}$, and $5.1 \%$ of the isolates had $\mathrm{IC}_{50}$ values above the cutoff value of $19 \mathrm{nM}$ for $\mathrm{NQ}$ (Figure 1). For the antifolate drug $\mathrm{PY}, \mathrm{IC}_{50} \leq 100$ $\mathrm{nM}, 100<\mathrm{IC}_{50} \leq 2000 \mathrm{nM}$, and $\mathrm{IC}_{50} \geq 2000 \mathrm{nM}$ were classified as sensitive, moderately resistant, and highly resistant. ${ }^{30,33}$ According to this classification scheme, all isolates tested were considered highly resistant to $P Y\left(I_{50} s \geq 2000 \mathrm{nM}\right)$ (Figure 1).

Temporal trend of in vitro drug sensitivities. There appeared to be three types of temporal trends of parasites' sensitivities to the seven antimalarials tested (Figure 2). For $\mathrm{CQ}$, there was a continuous decrease in mean $\mathrm{IC}_{50}$ values in 2012 , 2014, and 2016. The annual mean $\mathrm{IC}_{50}$ S for another 4aminoquinoline drug NQ and PND also showed a similar decreasing trend during 2010-2016. Interestingly, in vitro $I_{50} S$ of the parasites to the three aminoalcohol drugs MFQ, QN, and LMF showed a similar trend of fluctuation with a significant decrease in 2014 and an increase in 2016. Among them, parasites showed a significant higher $\mathrm{IC}_{50}$ to $\mathrm{LMF}$ and $\mathrm{QN}$ in 2016 than 2014 ( $P<0.01$, Mann-Whitney U test) (Figure 2). By contrast, only the $\mathrm{IC}_{50}$ values to PY showed a significant upward annual trend, with the $\mathrm{IC}_{50}$ values in 2014 and 2016 being significantly higher than the values in 2010 and $2012(P<$ 0.001).

Polymorphisms of drug resistance genes. We genotyped pfcrt, pfmdr1, pfdhps, and pfdhfr genes in the isolates collected in 2014 and 2016. For pfcrt, mutations at the M74l, 

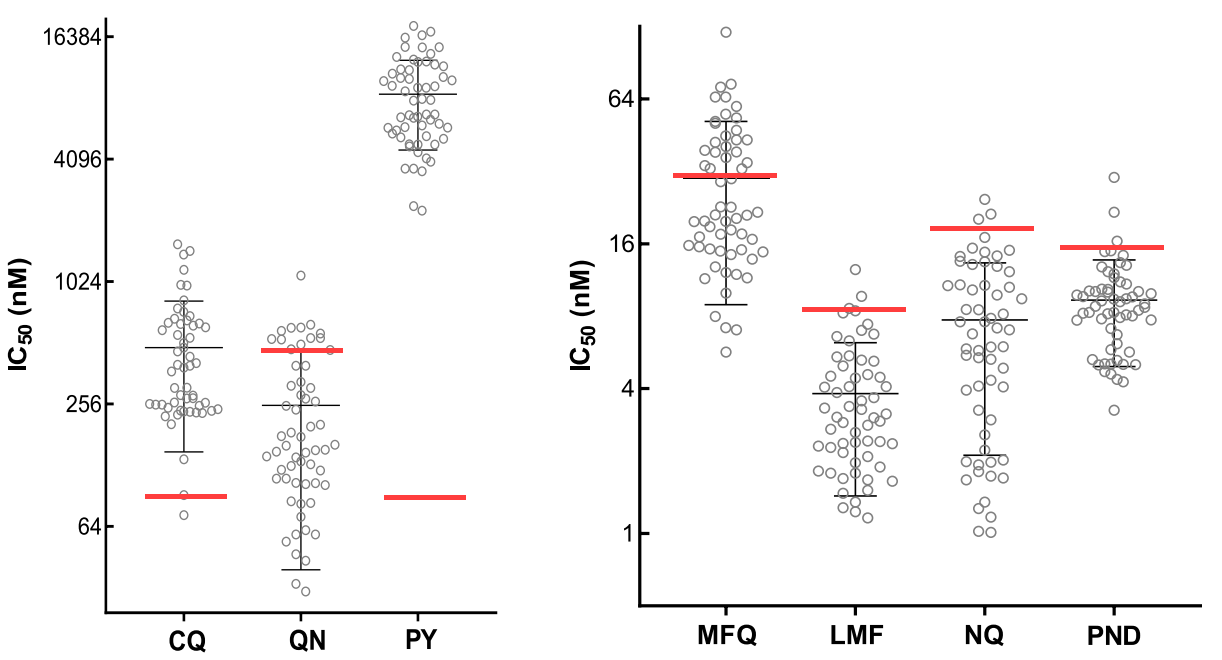

FIGURE 1. In vitro $I_{50}$ values of culture-adapted parasite isolates collected from the China-Myanmar border to seven antimalarial drugs chloroquine (CQ), quinine (QN), pyrimethamine (PY), mefloquine (MFQ), lumefantrine (LMF), naphthoquine (NQ), and pyronaridine (PND). The dot plots show the median and interquartile range. Red bars indicate the cutoff values used to define resistance: $C Q, 100 \mathrm{nM} ; \mathrm{QN}, 600 \mathrm{nM}$; PY, $100 \mathrm{nM}$; MFQ, $30 \mathrm{nM}$; LMF, $9.6 \mathrm{nM}$; NQ, $19 \mathrm{nM}$; and PND, $15 \mathrm{nM}$. Note that except for CQ and PY, the cutoff values for other drugs are arbitrary and do not necessarily correspond to in vivo clinical resistance. This figure appears in color at www.ajtmh.org.

N75E, K76T, A220S, Q271E, N326S, and R371I positions were all fixed in these isolates. Only one of the 15 parasites genotyped in 2014 was wild type at the I356T position (Table 2). For the pfmdr1 gene, the N86Y mutation was rare and found only in one isolate in 2014. Interestingly, the Y184F mutation was highly abundant in 2014 (86.7\%), whereas it was relatively infrequent in other years $(7.7-15 \%)\left(P<0.0001, x^{2}\right.$ test). Whereas the pfmdr1 1042 and 1246 positions all remained wild type, the $\mathrm{F} 1226 \mathrm{Y}$ mutation was identified in 2016 in 80\% (16/20) parasite isolates. For the pfdhps gene, the K540E/N mutations were fixed in samples from 2010, 2014, and 2016. Whereas the A581G mutation showed an increasing trend from $40 \%$ in 2010 to $95 \%$ in 2016 , the S436A mutation showed an opposite trend, decreasing from $40 \%$ in 2010 to disappearance in $2016(P<0.001)$. For the pfdhfr gene, both the N51I and I164L mutations were increased in frequency from 2010 and reached fixation in 2014 and 2016 $(P<0.05)$, whereas the $\mathrm{C} 59 \mathrm{R}$ and $\mathrm{S} 108 \mathrm{~N}$ mutations were fixed in all samples.

Association of mutations with in vitro drug susceptibilities. With the identification of the pfmdr1 F1226Y mutation in parasite samples from 2016, we wanted to determine whether the pfmdr1 mutations were associated with altered drug sensitivities. Neither Y184F nor F1226Y was associated with in vitro sensitivities of the parasites to $\mathrm{CQ}, \mathrm{NQ}$, or PY (data not shown). However, the Y184F mutation was associated with significantly increased sensitivities to the aminoalcoholic drugs QN $(P<0.001)$, LMF $(P<0.01)$, and MFQ $(P<0.05)$ as well as PND $(P<0.05)$ (Figure 3$)$. The F1226Y mutation was associated with significantly reduced sensitivities to QN $(P<0.001)$ and LMF $(P<0.05)$.

Haplotypes of drug resistance genes. For pfcrt, $98.2 \%$ of the parasite isolates carry the octuple mutations at the $74 / 75 / 76 / 220 / 271 / 326 / 356 / 371$ amino acid positions, a CQ-resistant genotype similar to that of Dd2 (Table 3). Three haplotypes were identified for the pfmdr1 gene at the $86 / 184 / 1226$ positions: the wild type (NYY) was predominant in 2010 and 2012, whereas two haplotypes with a single mutation NFF and NYY were the predominant haplotypes in 2014 and 2016, respectively (Table 3). For the antifolate resistance genes, $96.7 \%$ of the parasite isolates carried triple or quadruple mutations at the 521/59/108/164 positions in pfdhfr, although $88.2 \%$ of the parasite isolates carried a single or double mutation at $436 / 540 / 581$ positions in pfdhps. The quadruple mutant haplotype IRNL of $p f d h f r$ was present in $50 \%$ of the isolates in 2010 , as compared with complete fixation in 2014 and $2016(P<0.0001)$. By comparison, the double-mutation haplotypes (AE/NA and SE/ NG) in pfdhps, which were prevalent in 2010 and 2012, were

TABLE 1

In vitro $\mathrm{IC}_{50} \mathrm{~S}(\mathrm{nM})$ of culture-adapted Plasmodium falciparum isolates collected in 2014 and 2016 from the China-Myanmar border to seven antimalarial drugs

\begin{tabular}{|c|c|c|c|c|c|}
\hline \multirow[b]{2}{*}{ Drug } & \multicolumn{2}{|c|}{$2014(N=16)$} & \multicolumn{2}{|c|}{$2016(N=20)$} & \multirow[b]{2}{*}{$3 \mathrm{D} 7$ control (mean $\pm \mathrm{SD}$ ) } \\
\hline & Median (IQR) & Range & Median (IQR) & Range & \\
\hline Chloroquine* & $386.1(236.3-632.9)$ & $72.7-826.9$ & $254.0(235.7-408.9)$ & $222.6-727.0$ & $17.8 \pm 8.1$ \\
\hline Mefloquine & $16.9(12.6-19.9)$ & $7.2-29.8$ & $17.6(14.6-30.2)$ & $10.0-51.6$ & $19.9 \pm 1.2$ \\
\hline Lumefantrine & $1.9(1.6-2.3)$ & $1.2-3.0$ & $3.5(2.5-4.4)$ & $1.3-12.5$ & $1.5 \pm 0.2$ \\
\hline Quinine & $93.3(59.1-128.6)$ & 43.3-197.7 & $149.4(108.5-198.1)$ & $58.4-314.1$ & $34.6 \pm 1.7$ \\
\hline Naphthoquine & $5.6(1.9-7.8)$ & $1.0-14.8$ & $4.1(1.8-10.7)$ & $1.0-17.0$ & $7.5 \pm 0.5$ \\
\hline Pyrimethamine & $9705.0(6137.0-14256.0)$ & $4839.0-16679.0$ & $11021.0(9229.0-12592.0)$ & $6737.0-18522.0$ & $67.5 \pm 12.8$ \\
\hline Pyronaridine & $7.5(5.1-9.6)$ & $4.6-11.6$ & $7.9(5.4-10.3)$ & $3.3-13.4$ & $5.0 \pm 0.5$ \\
\hline
\end{tabular}

IQR = interquartile range (Mann-Whitney $\mathrm{U}$ test).

* For chloroquine, 15 isolates were tested in 2014 and 17 in 2016. 

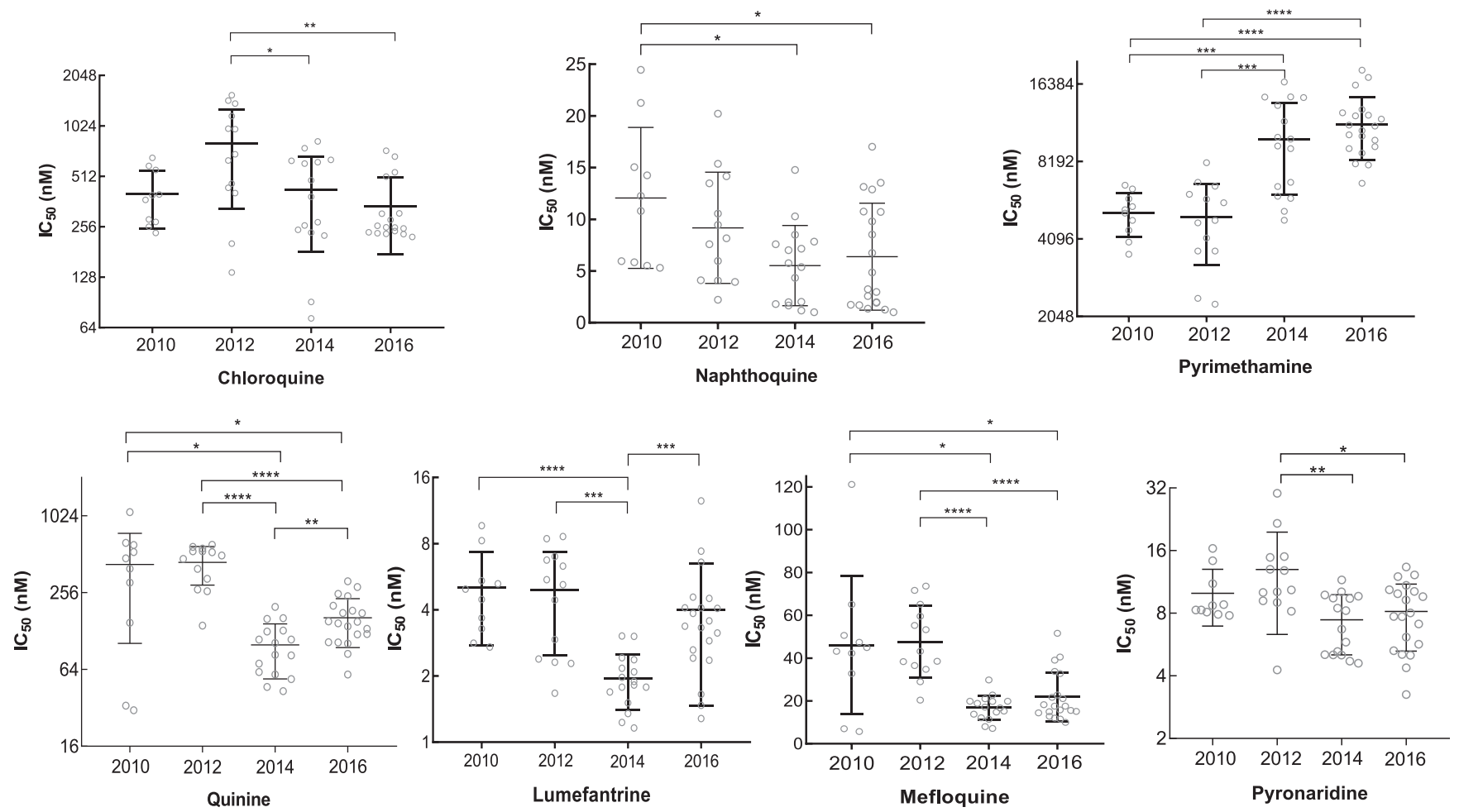

FIGURE 2. In vitro $I C_{50}$ values of culture-adapted parasite isolates from $2010,2012,2014$ and 2016 to seven antimalarial drugs. The dot plot shows that the median and interquartile range. ${ }^{*},{ }^{* *},{ }^{* \star *}$, and ${ }^{* \star * *}$ indicate significant difference between the years at $P<0.05,0.01,0.001$, and 0.0001 , respectively. Data for the 2010 and 2012 parasites were generated in a previous study. ${ }^{26}$

replaced by the single-mutation haplotype (SE/NA) in 2014 and 2016.

\section{DISCUSSION}

Plasmodium falciparum accounts for the vast majority of malaria-associated morbidity and mortality worldwide. The spread of multidrug-resistant $P$. falciparum is one of the most important factors hindering the global elimination of malaria. As the GMS is moving toward malaria elimination, the heavy malaria burden in Myanmar needs particular attention. Furthermore, with Myanmar's geographical connection to South Asia, drug resistance could be amplified here and spilled over to the Indian subcontinent. In this study, we further profiled

TABLE 2

Prevalence of non-synonymous mutations in drug resistance-associated genes in 2010-2016

\begin{tabular}{|c|c|c|c|c|c|c|c|}
\hline \multirow[b]{2}{*}{ Gene } & \multirow[b]{2}{*}{ Amino acid position } & \multicolumn{5}{|c|}{ Prevalence of isolates ( $n[\%])$} & \multirow[b]{2}{*}{$P$-value $\neq$} \\
\hline & & $2010(n=10)$ & $2012(n=13)$ & $2014(n=16)$ & $2016(n=20)$ & Total $(n=59)$ & \\
\hline \multirow[t]{8}{*}{ Pfcrt $^{\star}$} & M74I & $10(100)$ & $13(100)$ & $15(100)$ & $17(100)$ & $55(100)$ & ND \\
\hline & N75E & $10(100)$ & $13(100)$ & $15(100)$ & $17(100)$ & $55(100)$ & ND \\
\hline & K76T & $10(100)$ & $13(100)$ & $15(100)$ & $17(100)$ & $55(100)$ & ND \\
\hline & A220S & $10(100)$ & $13(100)$ & $15(100)$ & $17(100)$ & $55(100)$ & ND \\
\hline & Q271E & $10(100)$ & $13(100)$ & $15(100)$ & 17 (100) & 55 (100) & ND \\
\hline & N326S & $10(100)$ & $13(100)$ & $15(100)$ & $17(100)$ & $55(100)$ & ND \\
\hline & I356T & $10(100)$ & $13(100)$ & 14 (93.3) & $17(100)$ & 54 (98.2) & 0.438 \\
\hline & R371I & $10(100)$ & $13(100)$ & $15(100)$ & $17(100)$ & 55 (100) & ND \\
\hline \multirow[t]{3}{*}{ Pfmdr1† } & N86Y & 0 & 0 & $1(6.7)$ & 0 & 1 (1.7) & 0.405 \\
\hline & Y184F & 1 (10) & $1(7.7)$ & $13(86.7)$ & $3(15.0)$ & $18(31)$ & $<0.0001$ \\
\hline & F1226Y & 0 & 0 & 0 & $16(80.0)$ & $16(27.6)$ & $<0.0001$ \\
\hline \multirow[t]{4}{*}{ P. falciparum dihydrofolate reductase } & N51I & $7(70)$ & 10 (76.9) & $16(100)$ & 20 (100) & 53 (89.8) & 0.013 \\
\hline & C59R & $10(100)$ & $13(100)$ & 16 (100) & 20 (100) & 59 (100) & ND \\
\hline & S108N & 10 (100) & 13 (100) & $16(100)$ & 20 (100) & 59 (100) & ND \\
\hline & I164L & $8(80)$ & $8(61.5)$ & 16 (100) & 20 (100) & $52(88.1)$ & 0.003 \\
\hline \multirow[t]{3}{*}{ P. falciparum dihydropteroate synthase } & S436A & $4(40)$ & $4(30.8)$ & 1 (6.3) & 0 & $9(15.3)$ & 0.008 \\
\hline & K540E/N & $10(100)$ & 8 (61.5) & $16(100)$ & 20 (100) & 54 (91.5) & $<0.0001$ \\
\hline & A581G & $4(40)$ & 9 (69.2) & 15 (93.8) & 19 (95) & 47 (79.7) & 0.001 \\
\hline
\end{tabular}

The 2010 and 2012 data from Bai et al. ${ }^{26}$ were included for comparison. Mutations are shown in bold. ND = not done.

*Plasmodium falciparum chloroquine-resistant transporter (Pfcrt) gene was genotyped in 15 isolates in 2014 and 17 isolates in 2016.

† Plasmodium falciparum multidrug resistance 1 (Pfmdr1) gene was genotyped in 15 isolates in 2014.

$\ddagger \chi^{2}$ test comparison for each mutation among the 4 years. 


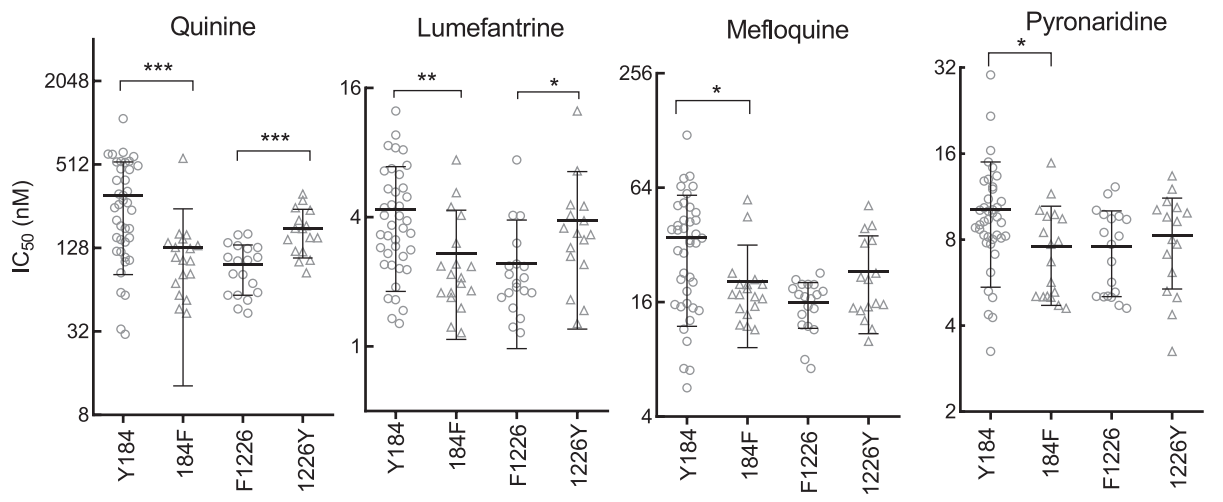

FIGURE 3. Association of the pfmdr1 Y184F and F1226Y mutations with in vitro sensitivities to quinine, lumefantrine, mefloquine, and pyronaridine in parasites collected from 2010 to 2016. ${ }^{*},{ }^{\star *}$, and ${ }^{* * *}$ indicate significance at $P<0.05,0.01$, and 0.001 , respectively.

in vitro drug sensitivities of parasite isolates obtained in recent years from the China-Myanmar border and genotyped molecular markers of antimalarial drug resistance in these isolates. Together with the drug sensitivity and molecular epidemiological data from earlier years, ${ }^{26,34}$ we provide detailed trends of drug resistance of the parasites from this region.

The drug sensitivity profile of the $P$. falciparum parasites may well reflect the local antimalarial drug policies. In Myanmar, ACTs (artemether-lumefantrine, DHA-PPQ, and ASMFQ) were introduced in 2008 as the first-line treatment for uncomplicated $P$. falciparum malaria. ${ }^{35}$ In the ChinaMyanmar border area, the predominant ACT used is DHA$P P Q$, and earlier studies indicated that DHA-PPQ retained excellent efficacy for uncomplicated $P$. falciparum malaria. ${ }^{36,37}$ It is noteworthy that despite recommendations from the national malaria treatment guidelines, various antimalarial drugs including artemisinin monotherapies could be found in the private sectors. Therefore, although all parasites evaluated in this study were collected after the introduction of ACTs, the extensive uses of other antimalarials may underline the dynamic changes of drug sensitivities of the parasites observed in our study area over the years.

Chloroquine resistance was discovered on the CambodiaThailand border as early as in the late 1950s, and resistant parasite strains spread to countries in Southeast Asia in the late
1960 s. $^{38}$ As we have found previously, ${ }^{26}$ parasite isolates in recent years remained highly resistant to $C Q$, a phenomenon that is often attributed to the wide use of $C Q$ for treating sympatric Plasmodium vivax infections. This in vitro drug susceptibility result is further supported by the molecular study of the pfcrt gene, which showed that $98.2 \%$ of the parasites carried the octuple mutations in the pfort gene. This particular Dd2-like pfcrt genotype is the most predominant genotype present in the GMS, and from this genetic background, the artemisinin and PPQ resistance has evolved. ${ }^{39,40}$ Despite the persistence of the Dd2-like pfcrt genotype, compared with 2012, there was a significant reduction in $\mathrm{IC}_{50}$ to $\mathrm{CQ}$ among parasites collected in 2014 and 2016, although all except two parasite isolates still had in vitro $\mathrm{IC}_{50}$ values above the $100 \mathrm{nM}$ cutoff value for highlevel resistance (Figure 2). We speculate that this change may be mediated by changes in other molecular markers such as pfmdr1 that modify CQ resistance. Despite that another 4aminoquinoline drug NQ showed a similar trend of reduction in $\mathrm{IC}_{50}$ after the year 2010, parasites remained sensitive to NQ with most of the parasites in 2014-2016 having $\mathrm{IC}_{50}$ values below 15 nM. In support of this, single-dose NQ in combination with azithromycin has been shown to be highly effective in preventing malaria infections as a monthly malaria prophylaxis drug in the China-Myanmar border area. ${ }^{41}$ This suggests that the major resistance mechanisms of $\mathrm{CQ}$ and $\mathrm{NQ}$ might be

TABLE 3

Prevalence of major haplotypes of drug resistance-associated genes in $P$. falciparum isolates from different years

\begin{tabular}{|c|c|c|c|c|c|c|c|}
\hline \multirow[b]{2}{*}{ Gene } & \multirow[b]{2}{*}{ Haplotypeł } & \multicolumn{5}{|c|}{ Prevalence of isolates $(n[\%])$} & \multirow[b]{2}{*}{$P$-value§ } \\
\hline & & $2010(n=10) \dagger$ & $2012(n=13) \dagger$ & $2014(n=16)$ & $2016(n=20)$ & Total $(n=59)$ & \\
\hline Pfcrt $^{*}$ & IETSESTI & $10(100)$ & $13(100)$ & $14(93.3)$ & $17(100)$ & $54(98.2)$ & 0.438 \\
\hline \multirow[t]{3}{*}{ Pfmdr1* } & NYF & $9(90)$ & $12(92.3)$ & $1(6.7)$ & $1(5)$ & $23(39.7)$ & $<0.0001$ \\
\hline & NFF & $1(10)$ & $1(7.7)$ & $13(86.7)$ & $3(15.0)$ & $18(31)$ & $<0.0001$ \\
\hline & NYY & - & - & - & $16(80.0)$ & $16(27.6)$ & $<0.0001$ \\
\hline \multirow[t]{3}{*}{ Pfdhfr } & IRNI & $2(20)$ & $3(23.1)$ & - & - & $5(8.5)$ & 0.035 \\
\hline & NRNL & $3(30)$ & $1(7.7)$ & - & - & $4(6.8)$ & 0.011 \\
\hline & IRNL & $5(50)$ & $7(53.8)$ & $16(100)$ & $20(100)$ & $48(81.4)$ & $<0.0001$ \\
\hline \multirow[t]{3}{*}{ Pfdhps } & SE/NA & $2(20)$ & - & 15 (93.8) & $19(95)$ & $36(61.0)$ & $<0.0001$ \\
\hline & $\mathrm{AE} / \mathrm{NA}$ & $4(40)$ & $3(23.1)$ & - & - & 7 (11.9) & 0.003 \\
\hline & SE/NG & $3(30)$ & $5(38.5)$ & - & $1(5)$ & 9 (15.3) & 0.009 \\
\hline
\end{tabular}

Only haplotypes with prevalence $\geq 5 \%$ were included. Mutations are shown in bold

${ }^{*}$ The $P$. falciparum chloroquine-resistant transporter (pfcrt) gene was tested in 15 strains in 2014 and 17 strains in 2016, and 55 strains in total. The $P$. falciparum multidrug resistance 1 (pfmdr1) genes were tested in 15 strains in 2014, and 58 strains in total.

† Data for 2010 and 2012 were from Yao Bai et al. ${ }^{26}$

$\ddagger$ Haplotypes were based on amino acids at the positions pfcrt $(74,75,76,220,271,326,356$, and 371$)$ : pfmdr1 (86, 184, and 1226); P. falciparum dihydropteroate synthase (pfdhps) (436, 540,

$581)$, and $P$. falciparum dihydrofolate reductase (pfdhfr) $(51,59,108$, and 164).

$\S$ The $P$-value analyzes the overall trend between four years ( $x 2$ test, SPSS 20). 
different. ${ }^{42}$ Another drug that showed a similar trend to the two aminoquinoline drugs is PND, a drug that is used as an injection at the China-Myanmar border. It is not widely used, and parasites remained highly sensitive to this drug. Resistance mechanism to PND is not clear, but cross-resistance to aminoquinolines was generally not observed. ${ }^{43}$

Interestingly, in vitro sensitivities of longitudinally collected parasite isolates to the three aminoalcohol drugs QN, LMF, and MFQ showed a similar trend with a significant decline in $I_{50}$ S in 2014, but a subsequent increase in 2016. Overall, the mean $\mathrm{IC}_{50}$ levels of these drugs in 2014-2016 remained much lower than in 2010-2012. Plasmodium falciparum multidrug resistance 1 amplification has been associated with resistance to this family of drugs, and extensive use of MFQ has selected parasites with high copy numbers of pfmdr $1 .{ }^{20,28,44}$ The very low level of pfmdr1 amplification in this region as shown in our earlier studies ${ }^{27,45}$ could be due to the lack of MFQ use and deployment of $\mathrm{PPQ}$ either as monotherapy or in ACT with DHA, which selects against pfmdr1 gene amplification. ${ }^{24,25}$ Although such a temporal fluctuation of in vitro sensitivities to these three drugs was not clear, it might be related to the drug use changes. Artemether-lumefantrine has been recently introduced to the study area in addition to DHA-PPQ as the main ACT used in the study area. Correlations in in vitro sensitivities to these three drugs suggest similar resistance mechanisms, and cross-resistance need to be monitored.

Results from the in vitro drug assay of PY and genotyping the pfdhfr and pfdhps genes all suggest that parasites from this region maintained a high degree of resistance to antifolate drugs. All parasites tested showed $\mathrm{IC}_{50}$ s to PY above $2000 \mathrm{nM}$ (highly resistant), and moreover, the in vitro susceptibilities of the parasite isolates to PY kept decreasing during 2014-2016, although antifolate drugs have been withdrawn from treating malaria a long time ago. Accompanying this increasing trend in in vitro $\mathrm{IC}_{50}$, several resistance-conferring mutations in pfdhfr (N51I) and pfdhps (K540E/N and A581G) had drastically increased in frequency and were fixed or approached fixation in 2014 and 2016. We do not know the sources for the continuous selection on these antifolate resistance genes but speculate that it could be due to collateral selection from extensive use of antifolate drugs for bacterial infections.

In addition to the observed temporal increase in pfdhfr and pfdhps mutations, changes in two other genes are also worth noting. For pfmdr1, we detected the F1226Y mutation in $80 \%$ parasite isolates in 2016, all of them being the $\mathrm{N}_{86} \mathrm{Y}_{184} \mathbf{Y}_{1226}$ haplotype. A study from the Thailand-Myanmar border showed that $\mathrm{F} 1226 \mathrm{Y}$ was associated with reduced in vitro artemisinin sensitivity, ${ }^{46}$ suggesting that this change may be due to the widespread use of ACTs in recent years. It is also noteworthy that our earlier study found that all parasites collected in 2014-2016 contained the NN insertion at amino acids 136-137, the F446I mutation, or the new mutation G533S at the PfK13 gene. ${ }^{34}$ Thus, it is also possible that these parasites from 2014 to 2016 may represent newly introduced parasites with the migrant human populations escaping the Kachin civil war. ${ }^{47}$

The combination of in vitro drug sensitivity assay and molecular surveillance provides a comprehensive picture of antimalarial drug resistance. Unfortunately, the cutoff values for resistance of some drugs such as NQ and PND are not defined, and molecular markers for resistance to some drugs are not known. One limitation of this study is the relatively small sample size. In recent years, as malaria incidence has gone down drastically, collection of samples for laboratory culture has become difficult. Thus, future studies may need to include clinical efficacy studies, on-site ex vivo drug assays, and molecular epidemiology studies so that the drug resistance information can be used to inform timely changes of drug policy.

Received April 1, 2020. Accepted for publication May 18, 2020.

Published online June 22, 2020.

Acknowledgments: We thank all the patients for volunteering to participate in the study. This study was supported by a grant (U19AI089672) from the National Institute of Allergy and Infectious Diseases, National Institutes of Health. Z. Y. was supported by the National Science Foundation of China (31860604 and U1802286) and Major Science and Technology Projects of Yunnan Province (2018ZF0081). C. L., X. C., and Y. Z. were supported by Yunnan Applied Basic Research Projects-Union Foundation (2017FE468185, 2018FE001-190, and 2015FB034, respectively).

Authors' addresses: Siqi Wang, Shiling Xu, Jinting Geng, Yu Si, Hui Zhao, Xinxin Li, Qi Yang, Weilin Zeng, Zheng Xiang, Xi Chen, Yanmei Zhang, Cuiying Li, and Zhaoqing Yang, Department of Pathogen Biology and Immunology, Kunming Medical University, Kunming, China, E-mails: 535150908@qq.com, 1393268692@qq.com, g492431833@163.com, 419853138@qq.com, 18435226968@163.com, 15736992870@163.com, 1814018076@qq.com, z867120268@163.com, xiangz721@163.com, 17431524@qq.com, syh707212a@126.com, licuiying2002@126.com, and zhaoqingy92@hotmail.com. Myat Phone Kyaw, Myanmar Health Network Organization, Yangon, Myanmar, E-mail: kyaw606@gmail.com. Liwang Cui, Department of Internal Medicine, Morsani College of Medicine, University of South Florida, Tampa, FL, E-mail: liwangcui@usf.edu.

\section{REFERENCES}

1. WHO, 2019. World Malaria Report 2019. Geneva, Switzerland: World Health Organization.

2. Feng J, Zhang L, Huang F, Yin JH, Tu H, Xia ZG, Zhou SS, Xiao N, Zhou XN, 2018. Ready for malaria elimination: zero indigenous case reported in the People's Republic of China. Malar J 17: 315.

3. Menard D, Dondorp A, 2017. Antimalarial drug resistance: a threat to malaria elimination. Cold Spring Harb Perspect Med 7: a025619.

4. Noedl H, Se Y, Schaecher K, Smith BL, Socheat D, Fukuda MM, 2008. Evidence of artemisinin-resistant malaria in western Cambodia. N Engl J Med 359: 2619-2620.

5. Dondorp AM et al., 2009. Artemisinin resistance in Plasmodium falciparum malaria. N Engl J Med 361: 455-467.

6. Carrara VI et al., 2009. Changes in the treatment responses to artesunate-mefloquine on the northwestern border of Thailand during 13 years of continuous deployment. PLoS One 4: e4551.

7. Phyo AP et al., 2012. Emergence of artemisinin-resistant malaria on the western border of Thailand: a longitudinal study. Lancet 379: 1960-1966.

8. Ashley EA et al., 2014. Spread of artemisinin resistance in Plasmodium falciparum malaria. N Engl J Med 371: 411-423.

9. Huang $F$ et al., 2015. A single mutation in $\mathrm{K} 13$ predominates in southern China and is associated with delayed clearance of Plasmodium falciparum following artemisinin treatment. J Infect Dis 212: 1629-1635.

10. Leang R, Barrette A, Bouth DM, Menard D, Abdur R, Duong S, Ringwald $P, 2013$. Efficacy of dihydroartemisinin-piperaquine for treatment of uncomplicated Plasmodium falciparum and Plasmodium vivax in Cambodia, 2008 to 2010. Antimicrob Agents Chemother 57: 818-826.

11. Saunders DL, Vanachayangkul P, Lon C, 2014. Dihydroartemisininpiperaquine failure in Cambodia. N Engl J Med 371: 484-485.

12. Spring MD et al., 2015. Dihydroartemisinin-piperaquine failure associated with a triple mutant including kelch13 C580Y in Cambodia: an observational cohort study. Lancet Infect Dis 15: 683-691. 
13. Leang R et al., 2015. Evidence of Plasmodium falciparum malaria multidrug resistance to artemisinin and piperaquine in western Cambodia: dihydroartemisinin-piperaquine open-label multicenter clinical assessment. Antimicrob Agents Chemother 59: 4719-4726.

14. Amaratunga $C$ et al., 2016. Dihydroartemisinin-piperaquine resistance in Plasmodium falciparum malaria in Cambodia: a multisite prospective cohort study. Lancet Infect Dis 16: 357-365.

15. Djimde A et al., 2001. A molecular marker for chloroquineresistant falciparum malaria. N Engl J Med 344: 257-263.

16. Ecker A, Lehane AM, Clain J, Fidock DA, 2012. PfCRT and its role in antimalarial drug resistance. Trends Parasitol 28: 504-514.

17. Dhingra SK et al., 2017. A variant PfCRT isoform can contribute to plasmodium falciparum resistance to the first-line partner drug piperaquine. mBio 8: e00303-e00317.

18. Ross LS et al., 2018. Emerging southeast Asian PfCRT mutations confer Plasmodium falciparum resistance to the first-line antimalarial piperaquine. Nat Commun 9: 3314 .

19. Sanchez CP, Dave A, Stein WD, Lanzer M, 2010. Transporters as mediators of drug resistance in Plasmodium falciparum. Int $J$ Parasitol 40: 1109-1118.

20. Pickard AL, Wongsrichanalai C, Purfield A, Kamwendo D, Emery K, Zalewski C, Kawamoto F, Miller RS, Meshnick SR, 2003. Resistance to antimalarials in southeast Asia and genetic polymorphisms in pfmdr1. Antimicrob Agents Chemother 47: 2418-2423.

21. Gregson A, Plowe CV, 2005. Mechanisms of resistance of malaria parasites to antifolates. Pharmacol Rev 57: 117-145.

22. Ariey $\mathrm{F}$ et al., 2014. A molecular marker of artemisinin-resistant Plasmodium falciparum malaria. Nature 505: 50-55.

23. Ménard D et al., 2016. A worldwide map of Plasmodium falciparum K13-propeller polymorphisms. $N$ Engl $J$ Med 374: 2453-2464.

24. Amato R et al., 2017. Genetic markers associated with dihydroartemisinin-piperaquine failure in Plasmodium falciparum malaria in Cambodia: a genotype-phenotype association study. Lancet Infect Dis 17: 164-173.

25. Witkowski B et al., 2017. A surrogate marker of piperaquineresistant Plasmodium falciparum malaria: a phenotypegenotype association study. Lancet Infect Dis 17: 174-183.

26. Bai $Y$ et al., 2018. Longitudinal surveillance of drug resistance in Plasmodium falciparum isolates from the China-Myanmar border reveals persistent circulation of multidrug resistant parasites. Int J Parasitol Drugs Drug Resist 8: 320-328.

27. Meng H, Zhang R, Yang H, Fan Q, Su X, Miao J, Cui L, Yang Z, 2010. In vitro sensitivity of Plasmodium falciparum clinical isolates from the China-Myanmar border area to quinine and association with polymorphism in the $\mathrm{Na}+\mathrm{H}+$ exchanger. Antimicrob Agents Chemother 54: 4306-4313.

28. Price RN et al., 2004. Mefloquine resistance in Plasmodium falciparum and increased pfmdr1 gene copy number. Lancet 364 : 438-447.

29. Garg S, Saxena V, Kanchan S, Sharma P, Mahajan S, Kochar D, Das A, 2009. Novel point mutations in sulfadoxine resistance genes of Plasmodium falciparum from India. Acta Trop 110: 75-79.

30. Ringwald $P$, Bickii J, Basco LK, 1996. In vitro activity of antimalarials against clinical isolates of Plasmodium falciparum in Yaounde, Cameroon. Am J Trop Med Hyg 55: 254-258.

31. Pradines B, Tall A, Parzy D, Spiegel A, Fusai T, Hienne R, Trape JF, Doury JC, 1998. In-vitro activity of pyronaridine and amodiaquine against African isolates (Senegal) of Plasmodium falciparum in comparison with standard antimalarial agents. J Antimicrob Chemother 42: 333-339.
32. Pradines B, Tall A, Fusai T, Spiegel A, Hienne R, Rogier C, Trape JF, Le Bras J, Parzy D, 1999. In vitro activities of benflumetol against 158 Senegalese isolates of Plasmodium falciparum in comparison with those of standard antimalarial drugs. Antimicrob Agents Chemother 43: 418-420.

33. Aubouy A, Jafari S, Huart V, Migot-Nabias F, Mayombo J, Durand R, Bakary M, Le Bras J, Deloron P, 2003. DHFR and DHPS genotypes of Plasmodium falciparum isolates from Gabon correlate with in vitro activity of pyrimethamine and cycloguanil, but not with sulfadoxine-pyrimethamine treatment efficacy. J Antimicrob Chemother 52: 43-49.

34. Zhang J et al., 2019. In vitro susceptibility of Plasmodium falciparum isolates from the China-Myanmar border area to artemisinins and correlation with $\mathrm{K} 13$ mutations. Int $\mathrm{J}$ Parasitol Drugs Drug Resist 10: 20-27.

35. ACTwatch Group,Thein ST, Khin HSS, Thi A, 2017. Insights into the availability and distribution of oral artemisinin monotherapy in Myanmar: evidence from a nationally representative outlet survey. Malar J 16: 170.

36. Wang $Y$ et al., 2015. Clinical efficacy of dihydroartemisininpiperaquine for the treatment of uncomplicated Plasmodium falciparum malaria at the China-Myanmar border. Am J Trop Med Hyg 93: 577-583.

37. Liu $\mathrm{H}$ et al., 2015. In vivo monitoring of dihydroartemisininpiperaquine sensitivity in Plasmodium falciparum along the China-Myanmar border of Yunnan province, China from 2007 to 2013. Malar J 14: 47.

38. Wellems TE, Plowe CV, 2001. Chloroquine-resistant malaria. $J$ Infect Dis 184: 770-776.

39. Dhingra SK, Gabryszewski SJ, Small-Saunders JL, Yeo T, Henrich PP, Mok S, Fidock DA, 2019. Global spread of mutant pfcrt and its pleiotropic impact on Plasmodium falciparum multidrug resistance and fitness. MBio 10: e02731-18.

40. Miotto $O$ et al., 2015. Genetic architecture of artemisinin-resistant Plasmodium falciparum. Nat Genet 47: 226-234.

41. Yang H, Wang J, Liu H, Li X, Nie R, Li C, Wang H, Wang Q, Cao Y, Cui L, 2018. Randomized, double-blind, placebo-controlled studies to assess safety and prophylactic efficacy of naphthoquineazithromycin combination for malaria prophylaxis in southeast Asia. Antimicrob Agents Chemother 62: e00793-18.

42. Moore BR, Laman M, Salman S, Batty KT, Page-Sharp M, Hombhanje F, Manning L, Davis TM, 2016. Naphthoquine: an emerging candidate for artemisinin combination therapy. Drugs 76: 789-804.

43. Croft SL, Duparc S, Arbe-Barnes SJ, Craft JC, Shin CS, Fleckenstein L, Borghini-Fuhrer I, Rim HJ, 2012. Review of pyronaridine anti-malarial properties and product characteristics. Malar J 11: 270.

44. Sidhu AB, Uhlemann AC, Valderramos SG, Valderramos JC, Krishna S, Fidock DA, 2006. Decreasing pfmdr1 copy number in Plasmodium falciparum malaria heightens susceptibility to mefloquine, lumefantrine, halofantrine, quinine, and artemisinin. J Infect Dis 194: 528-535.

45. Wang Z et al., 2012. In vitro sensitivity of Plasmodium falciparum from China-Myanmar border area to major ACT drugs and polymorphisms in potential target genes. PLoS One 7: e30927.

46. Veiga MI, Ferreira PE, Jornhagen L, Malmberg M, Kone A, Schmidt BA, Petzold M, Bjorkman A, Nosten F, Gil JP, 2011. Novel polymorphisms in Plasmodium falciparum ABC transporter genes are associated with major ACT antimalarial drug resistance. PLoS One 6: e20212.

47. Zhou G, Lo E, Zhong D, Wang X, Wang Y, Malla S, Lee MC, Yang Z, Cui L, Yan G, 2016. Impact of interventions on malaria in internally displaced persons along the China-Myanmar border: 2011-2014. Malar J 15: 471. 